\title{
Building Blocks for Magnon Optics: Emission and Conversion of Short Spin Waves - Supplementary Material
}

Felix Groß, ${ }^{*}{ }^{\dagger}$ Mateusz Zelent, ${ }^{\dagger}$ Nick Träger, ${ }^{\dagger}$ Johannes Förster, ${ }^{\dagger}$ Umut T. Sanli, $^{\dagger}$ Robert Sauter, ${ }^{\dagger}$ Martin Decker, "Christian H. Back, "Markus Weigand, ${ }^{\S}$ Kahraman Keskinbora, ${ }^{\dagger}$ Gisela Schütz $^{\dagger}$ Maciej Krawczyk, ${ }^{\ddagger}$ and Joachim Gräfe, ${ }^{* \dagger}$

$\dagger$ †ax Planck Institute for Intelligent Systems, 70569 Stuttgart, Germany $\ddagger$ Faculty of Physics, Adam Mickiewicz University, Poznań, 61-614 Poznań, Poland \Technical University Munich, 85748 Garching, Germany $\S$ Helmholtz-Zentrum Berlin für Materialien und Energie, 12489 Berlin, Germany E-mail: fgross@is.mpg.de graefe@is.mpg.de

Additional supplementary material not included in this file consists of three 3D rendered spin wave movies of converter and emitter. 


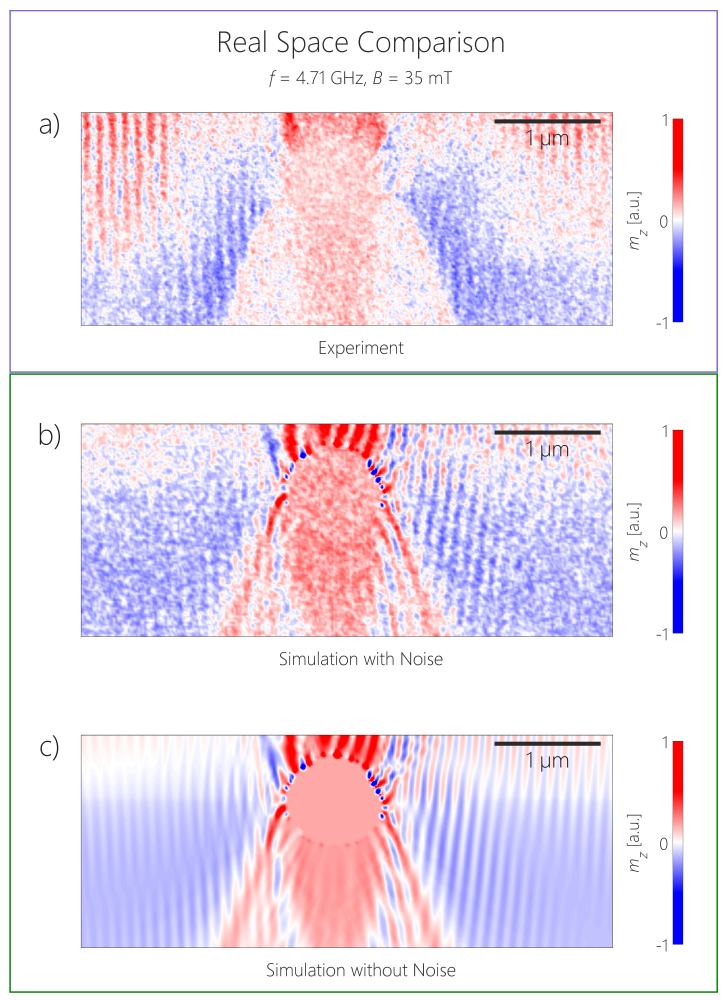

Figure 1: Real space comparison of experiment and simulation: a) Experimental data. b) Simulated data with added noise. c) Simulated data without noise. 


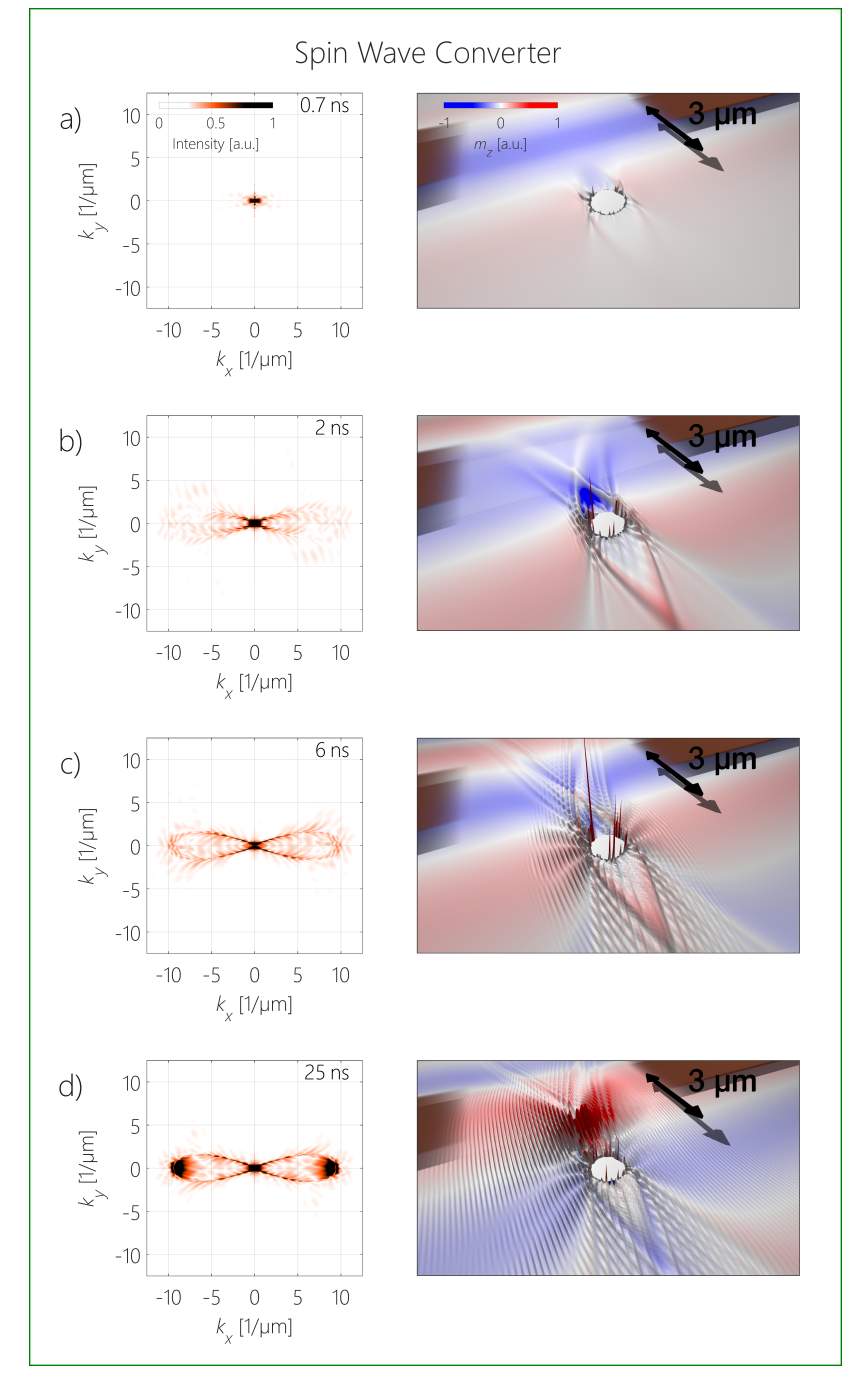

Figure 2: Simulated converter close to the microstrip antenna for different time frames.

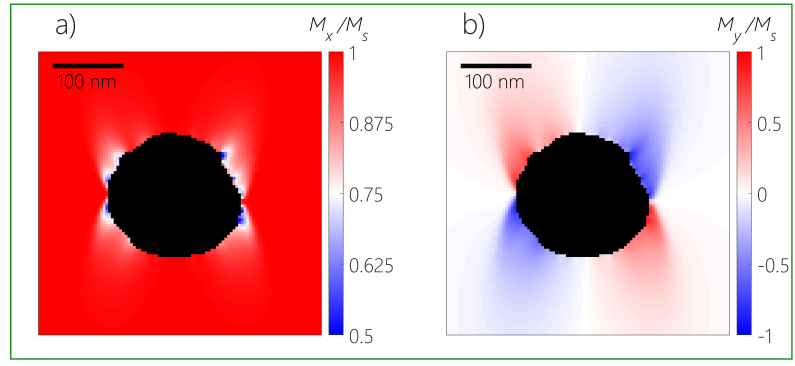

Figure 3: Simulated $x$ - and $y$-component of the static magnetization. Figure a) nicely displays the demagnetization structures responsible for the spin wave emission and conversion. 